\title{
Quantum Monte Carlo study of vibrational states of silanone
}

\author{
Paulo H. Acioli ${ }^{\mathrm{a}, *}$, L.S. Costa ${ }^{\mathrm{a}}$, Frederico V. Prudente ${ }^{\mathrm{b}}$ \\ ${ }^{\text {a }}$ Núcleo de Física Atômica, Molecular e de Fluidos Instituto de Física - Universidade de Brasília, CP 04455 , \\ 70919-970 Brasília - DF, Brazil \\ b Departamento de Química, Universidade de Coimbra, P-3049 Coimbra Codex, Portugal
}

Received 20 October 1999; in final form 23 December 1999

\begin{abstract}
We report correlation function quantum Monte Carlo (CFQMC) calculations of the vibrational levels on the silanone potential energy surface developed by Koput and co-workers. We computed the vibrational modes up to $3500 \mathrm{~cm}^{-1}$ and up to the fifth excited vibrational mode. Our results are in agreement with those reported by Koput et al. These results are important to future theoretical and experimental investigation of the $\mathrm{H}_{2} \mathrm{SiO}$ molecule, as spectroscopic data in this system are scarce. (C) 2000 Elsevier Science B.V. All rights reserved.
\end{abstract}

\section{Introduction}

The determination of the properties of small silicon-containing molecules has been the focus of attention of both experimental and theoretical studies [1-3]. These molecules and ions of decomposition $\left(\mathrm{Si}, \mathrm{O}, \mathrm{H}_{n}\right)$ may play an important role in interstellar chemistry [4]. One of the simplest molecules in this series, with a closed shell, is $\mathrm{H}_{2} \mathrm{SiO}$ (silanone). Thus, becoming one of the primary targets of study. The study of silanone is also of technical interest, as this molecule is predicted as a precursor of deposition for silicon dioxide films $[5,6]$.

However, as silanone is a highly reactive species, there has been only a few precise spectroscopic data, like the rotational spectrum in the millimeterwave range $[7,3]$. As a consequence of this lack of experi-

\footnotetext{
* Corresponding author. Fax: +55-61-307-2363; e-mail: pacioli@tatu.fis.unb.br
}

mental results, the theoretical determination of the rotational and vibrational spectra plays a very important role, and therefore a high quality potential energy surface (PES) is highly desirable. For many years, the theoretical study stayed centered on the determination of the equilibrium geometry, fundamentals and harmonic frequencies, and the anharmonic force fields of the $\mathrm{H}_{2} \mathrm{SiO}$ and its isomers, always using semi-empirical or ab initio electronic calculations [8-14].

Only recently, Koput and co-workers [15] have developed a full 6-dimensional potential energy surface and have computed some vibrational and rotational energy levels of silanone, $\mathrm{H}_{2} \mathrm{SiO}$. In particular, they have utilized the Coupled Cluster with singles and doubles corrected to triple excitations $(\operatorname{CCSD}(\mathrm{T}))$ with a correlation correction up to a quintuple-zeta basis set (cc-V5Z) [16,17] in order to calculate the $\mathrm{ab}$ initio points and they employed the Simons-Parr-Finlan (SPF) expansion up to sextic order [18] in order to fit the PES. 
In this work, we utilize the PES developed by Koput et al. [15] in order to compute the vibrational energy levels of silanone utilizing the correlation function quantum Monte Carlo (CFQMC) method $[19,20]$. The CFQMC method, like the other quantum Monte Carlo methods, is based on the stochastic principle [21] and has been successfully applied to the computation of excited rotational and vibrational energy levels of up to tetraatomic molecules [19,20,22-25]. We have computed the vibrational levels of $\mathrm{H}_{2} \mathrm{SiO}$ up to $3500 \mathrm{~cm}^{-1}$ and up to the fifth excited vibrational mode. Our results are comparable to the ones reported by Koput et al. using the perturbational and the variational approaches and to the Martin's results for fundamental frequencies [14].

This Letter is organized as follows, in Section 2 we give a short description of the CFQMC method, in Section 3 we present our results and discussion, the final section is dedicated to our concluding remarks.

\section{Correlation function quantum Monte Carlo}

To obtain the rovibrational energy levels is necessary to solve the following eigenvalue problem:

$H \Phi_{i}(\boldsymbol{R})=E_{i} \Phi_{i}(\boldsymbol{R})$,

where $H$ is the Hamiltonian of the system,

$H=-\sum_{i=1}^{N} \frac{\hbar^{2}}{2 m_{i}} \nabla_{i}^{2}+V(\boldsymbol{R})$,

$m_{i}$ is the mass of particle $i, \boldsymbol{R}$ is the vector of $3 N$ coordinates, $V(\boldsymbol{R})$ is the potential energy surface, and $E_{i}$ and $\Phi_{i}$ are the eigenvalues and eigenvectors of $H$, respectively.

Let $\left\{f_{\alpha}(\boldsymbol{R})\right\}$ be a trial basis set of $m$ known functions, we can define the overlap and Hamiltonian matrix elements as

$$
\begin{aligned}
& N_{\alpha \beta}(t)=\int \mathrm{d} \boldsymbol{R}_{1} \mathrm{~d} \boldsymbol{R}_{2} f_{\alpha}\left(\boldsymbol{R}_{2}\right) \mathrm{e}^{-t H} f_{\beta}\left(\boldsymbol{R}_{1}\right), \\
& H_{\alpha \beta}(t)=\int \mathrm{d} \boldsymbol{R}_{1} \mathrm{~d} \boldsymbol{R}_{2} H f_{\alpha}\left(\boldsymbol{R}_{2}\right) \mathrm{e}^{-t H} f_{\beta}\left(\boldsymbol{R}_{1}\right),
\end{aligned}
$$

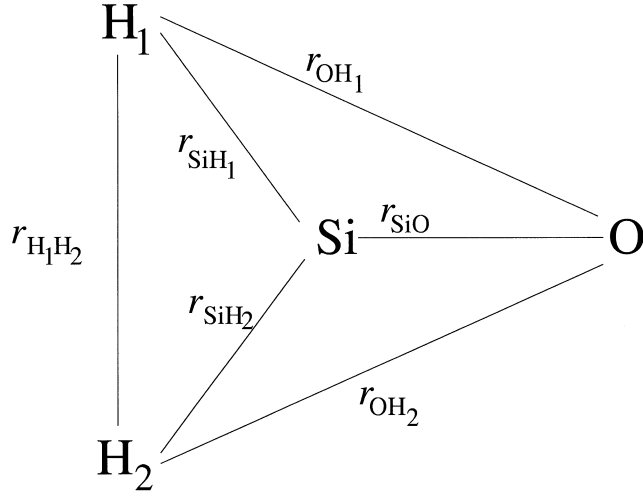

Fig. 1. Internal coordinates for $\mathrm{H}_{2} \mathrm{SiO}$ used in this work.

respectively. Associated with these matrices, the generalized eigenvalue problem is defined as

$$
\sum_{\beta=1}^{m}\left[H_{\alpha \beta}(t)-\Lambda_{k}(t) N_{\alpha \beta}(t)\right] d_{k \beta}(t)=0,
$$

with $d_{k}(t)$ being the $k$ th eigenvector and $\Lambda_{k}(t)$ its associated eigenvalue. It has been shown $[19,20]$ that

$$
\lim _{t \rightarrow \infty} \Lambda_{k}(t)=E_{k}, \quad 1 \leq k \leq m,
$$

as long as the basis set $\left\{f_{\alpha}(\boldsymbol{R})\right\}$ has some overlap with the eigenstates $\left\{\Phi_{i}\right\}$, and is linearly independent. The CFQMC consists of computing the matrix elements defined in Eq. (3) using Monte Carlo methods. In particular, we utilize two versions of the CFQMC method: the variational Monte Carlo version, which considers $t=0$ in Eqs. (3) and (4), and the diffusion Monte Carlo. As the purpose of this Letter is not the discussion of the method we refer the interested reader to Refs. [19,20,22-25].

The trial basis set utilized in this Letter is the one propose by Acioli and Soares Neto [24], which is a variation of the basis set proposed by Bernu et al. [20] to compute the vibrational levels of the water and formaldehyde molecules. The basis set is based on an harmonic approximation. For the ground state we use:

$\Psi_{0}=\exp \left(\sum_{\nu, \eta} \Delta S_{\nu} A_{\nu, \eta} \Delta S_{\eta}\right)$,

and for the excited states we use:

$$
\tilde{f}_{n_{1}, n_{2}, n_{3}, n_{4}, n_{5}, n_{6}}=\Psi_{0} \prod_{\nu=1}^{6}\left(\Delta S_{\nu}\right)^{n_{\nu}},
$$


Table 1

Variational parameters $\left\{A_{\nu, \mu}\right\}$ for the trial ground state wave function of $\mathrm{H}_{2} \mathrm{SiO}$

\begin{tabular}{lrrrrrr}
\hline$\left\{A_{\nu, \mu}\right\}$ & \multicolumn{1}{c}{$\mathrm{SiH}_{1}$} & \multicolumn{1}{c}{$\mathrm{SiH}_{2}$} & \multicolumn{1}{c}{$\mathrm{SiO}$} & $\mathrm{H}_{1} \mathrm{H}_{2}$ & \multicolumn{1}{c}{$\mathrm{OH}_{1}$} & $\mathrm{OH}_{2}$ \\
\hline $\mathrm{SiH}_{1}$ & -9.1269330 & 3.2013281 & 1.8619704 & 0.6371071 & -0.0794402 & -4.7393175 \\
$\mathrm{SiH}_{2}$ & 3.2013281 & -9.1269330 & 1.8619704 & 0.6371071 & -4.7393175 & -0.0794402 \\
$\mathrm{SiO}$ & 1.8619704 & 1.8619704 & -48.6702356 & 0.0217145 & -2.7337725 & -2.7337725 \\
$\mathrm{H}_{1} \mathrm{H}_{2}$ & 0.6371071 & 0.6371071 & 0.0217145 & -2.7043377 & 1.3523291 & 1.3523291 \\
$\mathrm{OH}_{1}$ & -0.0794402 & -4.7393175 & -2.7337725 & 1.3523291 & -1.8789047 & 5.0720539 \\
$\mathrm{OH}_{2}$ & -4.7393175 & -0.0794402 & -2.7337725 & 1.3523291 & 5.0720539 & -1.8789047 \\
$r_{\nu}^{0}$ & 2.7951500 & 2.7951500 & 2.8827200 & 4.6289500 & 3.6958800 & 3.6958800 \\
\hline
\end{tabular}

where $\Delta S_{\nu}=r_{i j}-r_{i j}^{0}, r_{i j}$ are the internuclear distances between the $i$ th and $j$ th atoms, and $r_{i}^{0}$ are their corresponding equilibrium values. We kept all the six internal modes in the ground state and excited

Table 2

Vibrational energy levels for the $\mathrm{H}_{2} \mathrm{SiO}$ molecule. DMC are the results of the present work. Variational are the results of Koput et al. [15] using the variational method. Perturbational are the results of Koput et al. [15] using the perturbational method. Martin are the results form Ref. [14]. Exp. is the experimental result for the $\nu_{2}$ vibrational mode from Ref. [26]. Energies are in $\mathrm{cm}^{-1}$

\begin{tabular}{|c|c|c|c|c|c|}
\hline Assignment & DMC & Variational & Perturbational & Martin [14] & Exp. [26] \\
\hline$\nu_{6}$ & 676.2 & 680.1 & 674.5 & 679.5 & - \\
\hline$\nu_{4}$ & 688.1 & 690.9 & 683.0 & 691.6 & 697 \\
\hline$\nu_{3}$ & 985.8 & 994.3 & 996.1 & 992.9 & - \\
\hline$\nu_{2}$ & 1210.8 & 1206.9 & 1200.5 & 1202.9 & 1202 \\
\hline $2 v_{6}$ & 1344.8 & 1358.3 & 1348.9 & - & - \\
\hline$\nu_{4}+\nu_{6}$ & 1369.6 & 1375.4 & 1349.8 & - & - \\
\hline $2 \nu_{4}$ & 1381.9 & 1383.8 & 1357.0 & - & - \\
\hline$\nu_{3}+\nu_{6}$ & 1666.8 & 1670.5 & 1666.6 & - & - \\
\hline$\nu_{3}+\nu_{4}$ & 1676.6 & 1684.2 & 1682.0 & - & - \\
\hline$\nu_{2}+\nu_{6}$ & 1883.0 & 1883.3 & 1871.4 & - & - \\
\hline$\nu_{2}+\nu_{4}$ & 1893.0 & 1893.7 & 1880.6 & - & - \\
\hline $2 \nu_{3}$ & 1985.3 & 1985.5 & 1989.4 & - & - \\
\hline $3 v_{6}$ & 2045.8 & - & - & - & - \\
\hline$\nu_{4}+2 \nu_{6}$ & 2058.3 & - & - & - & - \\
\hline $2 \nu_{4}+\nu_{6}$ & 2079.9 & - & - & - & - \\
\hline $3 v_{4}$ & 2089.4 & - & - & - & - \\
\hline$\nu_{1}$ & 2181.5 & 2171.0 & 2163.4 & 2162.0 & - \\
\hline$\nu_{5}$ & 2194.7 & 2191.3 & 2184.5 & 2186.2 & - \\
\hline$\nu_{2}+\nu_{3}$ & 2202.4 & 2202.8 & 2199.5 & 2198.4 & - \\
\hline$\nu_{3}+2 \nu_{6}$ & 2355.0 & - & - & - & - \\
\hline$\nu_{3}+\nu_{4}+\nu_{6}$ & 2371.0 & - & - & - & - \\
\hline$\nu_{3}+2 \nu_{4}$ & 2390.9 & - & - & - & - \\
\hline $2 \nu_{2}$ & 2407.0 & 2403.0 & 2391.0 & - & - \\
\hline$\nu_{2}+2 \nu_{6}$ & 2561.1 & - & - & - & - \\
\hline$\nu_{2}+\nu_{4}+\nu_{6}$ & 2569.7 & - & - & - & - \\
\hline$\nu_{2}+2 \nu_{4}$ & 2593.5 & - & - & - & - \\
\hline $2 \nu_{3}+\nu_{6}$ & 2659.7 & - & - & - & - \\
\hline $2 \nu_{3}+\nu_{4}$ & 2683.3 & - & - & - & - \\
\hline $4 \nu_{6}$ & 2729.0 & - & - & - & - \\
\hline$\nu_{4}+3 \nu_{6}$ & 2744.4 & - & - & - & - \\
\hline
\end{tabular}


trial functions. These internal modes are denoted by $r_{\mathrm{SiH}_{1}}, r_{\mathrm{SiH}_{2}}, r_{\mathrm{SiO}}, r_{\mathrm{OH}_{1}}, r_{\mathrm{OH}_{1}}$ and $r_{\mathrm{H}_{1} \mathrm{H}_{2}}$, according to Fig. 1. The variational parameters $\left\{A_{\nu \eta}\right\}$ are optimized in order to minimize the variational energy of the ground state $\left[n_{\nu}=0\right.$ in Eq. (7)] or its variance, within the framework of the standard variational Monte Carlo (VMC) method. Our choice of guiding function $\Psi$ to generate the random walks in the Metropolis sampling is

$$
\Psi=\left[\Psi_{0}\right]^{1 / \Gamma},
$$

where $\Gamma$ is chosen in order to assure a good integration of all states included in our calculation.

\section{Results}

In this section, we present the results of the CFQMC method for the computation of vibrational states of $\mathrm{H}_{2} \mathrm{SiO}$. For this calculation we optimized the parameters $\left\{A_{\nu, \mu}\right\}$ minimizing first the local energy associated with the ground state (guiding function). After this first optimization, we reoptimized the parameters by minimizing the variance of the local ground state energy. The optimized parameters are shown in Table 1.

For the excited state calculation, at the variational Monte Carlo level, we included all basis function such as $\sum_{i=1}^{6} n_{i} \leq 5$ in Eq. (7), in a total of 462 basis functions. We believe the variational basis functions to be good up to $3500 \mathrm{~cm}^{-1}$. At the diffusion Monte Carlo level, when we project the excited states from the improved trial basis set, we kept 60 states. In order to assign the quantum vibrational numbers we have taken the fundamental frequencies and we determined the classical energies in a classical fashion as $E\left(\nu_{1}, \nu_{2}, \nu_{3}, \nu_{4}, \nu_{5}, \nu_{6}\right)=\nu_{1} E_{1}+\nu_{2} E_{2}+\nu_{3} E_{3}+$ $\nu_{4} E_{4}+\nu_{5} E_{5}+\nu_{6} E_{6}$. Where $\nu_{1}$ corresponds to the symmetric $\mathrm{SiH}$ stretch mode, $\nu_{2}$ to the $\mathrm{SiO}$ stretch, $\nu_{3}$ to the symmetric $\mathrm{HSiO}$ bending mode, $\nu_{4}$ to the out-of-plane bending mode, $\nu_{5}$ asymmetric $\mathrm{SiH}$ stretch, and $\nu_{6}$ to the asymmetric $\mathrm{HSiO}$ bending mode. Our results obtained from the CFQMC calculations are displayed in Tables 2 and 3, along with the results of Koput et al. on the same PES using the traditional variational and perturbational approaches, the fundamental frequencies calculated by Martin and one experimental value $\left(\nu_{2}\right.$ fundamental fre-
Table 3

Vibrational energy levels for $\mathrm{H}_{2} \mathrm{SiO}$ molecule. DMC are the results of the present work. Variational are the results of Koput et al. [15] using the variational method. Perturbational are the results of Koput et al. [15] using the perturbational method. Energies are in $\mathrm{cm}^{-1}$

\begin{tabular}{|c|c|c|c|}
\hline Assignment & DMC & Variational & Perturbational \\
\hline $2 \nu_{4}+2 \nu_{6}$ & 2766.7 & - & - \\
\hline $3 \nu_{4}+\nu_{6}$ & 2793.8 & - & - \\
\hline $4 \nu_{4}$ & 2825.6 & - & - \\
\hline$\nu_{1}+\nu_{6}$ & 2836.0 & 2843.8 & 2836.4 \\
\hline$\nu_{1}+\nu_{4}$ & 2849.2 & 2855.0 & 2844.3 \\
\hline$\nu_{5}+\nu_{6}$ & 2863.7 & 2866.1 & 2853.9 \\
\hline$\nu_{2}+\nu_{3}+\nu_{6}$ & 2875.2 & - & - \\
\hline$\nu_{4}+\nu_{5}$ & 2880.1 & 2873.1 & 2858.0 \\
\hline$\nu_{2}+\nu_{3}+\nu_{4}$ & 2897.1 & - & - \\
\hline $3 \nu_{3}$ & 3030.2 & - & - \\
\hline$\nu_{3}+3 \nu_{6}$ & 3064.4 & - & - \\
\hline$\nu_{3}+\nu_{4}+2 \nu_{6}$ & 3069.3 & - & - \\
\hline$\nu_{3}+2 \nu_{4}+\nu_{6}$ & 3080.5 & - & - \\
\hline$\nu_{3}+3 \nu_{4}$ & 3096.6 & - & - \\
\hline $2 \nu_{2}+\nu_{6}$ & 3102.5 & - & - \\
\hline $2 \nu_{2}+\nu_{4}$ & 3127.2 & - & - \\
\hline$\nu_{1}+\nu_{3}$ & 3146.7 & 3152.6 & 3154.1 \\
\hline$\nu_{3}+\nu_{5}$ & 3164.2 & 3172.5 & 3167.8 \\
\hline$\nu_{2}+2 \nu_{3}$ & 3203.5 & - & - \\
\hline$\nu_{2}+3 \nu_{6}$ & 3239.9 & - & - \\
\hline$\nu_{2}+\nu_{4}+2 \nu_{6}$ & 3270.4 & - & - \\
\hline$\nu_{2}+2 \nu_{4}+\nu_{6}$ & 3290.3 & - & - \\
\hline$\nu_{2}+3 \nu_{4}$ & 3329.5 & - & - \\
\hline $2 \nu_{3}+2 \nu_{6}$ & 3361.7 & - & - \\
\hline $2 \nu_{3}+\nu_{4}+\nu_{6}$ & 3394.2 & - & - \\
\hline $2 \nu_{3}+2 \nu_{4}$ & 3407.5 & - & - \\
\hline$\nu_{1}+\nu_{2}$ & 3414.6 & 3370.5 & 3368.7 \\
\hline$\nu_{2}+\nu_{5}$ & 3424.9 & 3399.3 & 3386.4 \\
\hline $5 \nu_{6}$ & 3481.9 & - & - \\
\hline
\end{tabular}

quency) [26]. One can see that there is a reasonable agreement between our results and the results from the variational approach.

\section{Conclusion}

In this work, we report the use of CFQMC calculations of the vibrational modes of planar silanone $\left(\mathrm{H}_{2} \mathrm{SiO}\right)$. We present the eigenvalues of the planar vibrational modes, up to the fifth excited vibrational mode, up to $3500 \mathrm{~cm}^{-1}$. These results are of great importance, as $\mathrm{H}_{2} \mathrm{SiO}$ is highly reactive and experimental and theoretical results are scarce. Our results 
are in agreement with the results reported by Koput et al.

We are currently expanding our calculations to include the rotating wavefunctions proposed by Prudente et al. [23,25,27], to compute the rovibrational modes, up to $J=2$, for the silanone $\left(\mathrm{H}_{2} \mathrm{SiO}\right)$ molecule.

\section{Acknowledgements}

This work has been supported by Conselho Nacional de Desenvolvimento Científico e Tecnológico (CNPq-Brazil) and Coordenação de Aperfeiçoamento de Pessoal de Nível Superior (CAPES-Brazil) through grants to the authors. We also thank the Fundação de Apoio à Pesquisa - DF (FAP-DF) for computer facilities through grant 193.072/96.

\section{References}

[1] B.T. Luke, J.A. Pople, M.-B. Krogh-Jespersen, Y. Apeloig, M. Karni, J. Chandrasekhar, P. von Ragué Schleyer, J. Am. Chem. Soc. 108 (1986) 270.

[2] K. Raghavachari, J. Chandrasekhar, M.J. Frish, J. Am. Chem. Soc. 104 (1982) 5054.

[3] M. Bogey, B. Delcroix, A. Walters, J. Guillemin, J. Mol. Spectrosc. 175 (1996) 421.

[4] J.L. Turner, A. Dalgarno, Astrophys J 213 (1977) 386.

[5] G. Lucovsky, D. Tsu, R. Rudder, R Markunas, in: J.L.
Vossen, W. Kern (Eds.), Thin Filam Processes II, Academic, Boston, 1991, p. 55-619.

[6] M.J. Kushner, J. Appl. Phys. 74 (1993) 6538.

[7] S. Bailleaux, M. Bogey, C. Cemuynck, J.-L. Destombes, A. Walters, J. Chem. Phys. 101 (1994) 2719.

[8] T. Kudo, S. Nagase, J. Phys. Chem. 88 (1984) 2833.

[9] D.A. Dixon, J.L. Gole, Chem. Phys. Lett. 125 (1986) 179.

[10] S. Sakai, M.S. Gordon, K.D. Jordan, J. Phys. Chem. 92 (1988) 7053.

[11] M.S. Gordon, L.A. Pederson, J. Phys. Chem. 94 (1990) 5527.

[12] C.L. Darling, H.B. Schlegel, J. Phys. Chem. 97 (1993) 8207.

[13] B. Ma, H.H. Schaefer III, J. Chem. Phys. 101 (1994) 2734.

[14] J.M.L. Martin, J. Phys. Chem. A 102 (1998) 1394.

[15] J. Koput, S. Carter, N.C. Handy, Chem. Phys. Lett. 301 (1999) 1.

[16] T.H. Dunning Jr., J. Chem. Phys. 90 (1989) 1007.

[17] D.E. Woon, T.H. Dunning Jr., J. Chem. Phys. 98 (1993) 1358.

[18] G. Simons, R.G. Parr, J.M. Finlan, J. Chem. Phys. 59 (1973) 3229.

[19] D.M. Ceperley, B. Bernu, J. Chem. Phys. 89 (1988) 6316.

[20] B. Bernu, D.M. Ceperley, W.A. Lester Jr., J. Chem. Phys. 93 (1990) 553.

[21] B.L. Hammond, W.A. Lester Jr., P.J. Reynolds, in: Monte Carlo Methods in Ab Initio Quantum Chemistry, World Scientific, Singapore, 1994.

[22] F.V. Prudente, Paulo H. Acioli, J.J. Soares Neto. J. Chem. Phys. 109 (1998) 8801.

[23] F.V. Prudente, P.H. Acioli, Chem. Phys. Lett. 302 (1999) 249.

[24] P.H. Acioli, J.J. Soares Neto, J. Mol. Struct. 464 (1999) 145.

[25] P.H. Acioli, L.S. Costa, F.V. Prudente, J. Chem. Phys. 111 (1999) 6311.

[26] R. Withnall, L. Andrews, J. Phys. Chem. 89 (1985) 3261.

[27] L.S. Costa, F.V. Prudente, P.H. Acioli, Phys. Rev. A 6001 (2000) 2506. 